\title{
On some problems on composition of arithmetic functions
}

\author{
ILDIKÓ KÉZÉR
}

\begin{abstract}
The main goal of this paper is to investigate some problems related to the commutativity of the composition of arithmetic functions. The concept of commutativity arises many times in high school maths, so it is natural to study the composition of functions, namely the equation $f(g(n))=g(f(n))$, where $f$ and $g$ are such well known arithmetic functions as $d(n), \varphi(n), \sigma(n), \omega(n)$, or $\Omega(n)$. We study various aspects of solvability: can we exhibit infinitely many solutions; can we determine every solution; can we find suitable values in the range of both functions $f$ and $g$ for which the equation is, or is not solvable, respectively. We need just the basic facts about the above functions, and we use only elementary methods in the proofs. We present some interesting questions, their solutions, and raise some unsolved problems. We found that this topic can be discussed well in secondary school, mainly within the framework of group study sessions as we had some classes with a group of kids in 9th grade. We summarize the experiences of this experiment in the last section.
\end{abstract}

Key words and phrases: arithmetic functions, composition, commutativity.

ZDM Subject Classification: F60.

\section{Introduction}

The concept of commutativity occurs many times in high school maths, moreover, even earlier, in primary school maths. A really important situation arises in connection with the composition of functions. If we talk about the composition of geometric transformations or ordinary functions and note that composition is always associative, it is natural to ask if there are any non-trivial pairs of functions $f$ and $g$, for which $f(g(x))=g(f(x))$ holds for every $x$ in the domain. For 
instance can the order of two reflections be changed without the result being different? Or: we call students' attention not to make the mistake $-x^{2}=(-x)^{2}$. But of course they can find for example $f(x)=|x|$ and $g(x)=x^{2}$, for which $f(g(x))=g(f(x))$ is true for all $x \in \mathbb{R}$. We do require commutativity, as well, when we teach the concept of the inverse of a function, as the inverse of function $f$ is function $g$ iff $g(f(x))=f(g(x))=x$ for all $x \in \mathcal{D}_{f} \cap \mathcal{D}_{g}$ (where $\mathcal{D}_{f}$ denotes the domain of function $f$ ).

It is natural to ask after studying the question of commutativity for ordinary functions or geometric transformations, what can we say about the composition of some well known arithmetic functions. We have simple formulas for these, while their behaviors are multifarious. We will study the equation $f(g(n))=g(f(n))$ from different aspects such as: can we characterize all solutions, can we have infinitely many solutions, or can we find one solution at least. We will study also the equation $f(g(n))=g(f(n))=k$, for a fixed $k$, where $k \in \mathcal{R}_{f} \cap \mathcal{R}_{g}$, asking the same questions $\left(\mathcal{R}_{f}\right.$ denotes the range of function $\left.f\right)$.

We note that there are many papers investigating the composition of arithmetic functions from other aspects (see e.g. [4]-[8]), but our questions and results seem to have no precedents.

We work only with the following arithmetic functions: $d(n)$, which denotes the number of divisors of $n ; \sigma(n)$, which denotes the sum of divisors of $n ; \varphi(n)$, which denotes the number of positive integers up to $n$ relatively prime to $n ; \omega(n)$, which denotes the number of different prime factors of $n$; and $\Omega(n)$, which denotes the number of all prime factors of $n$ (counted with multiplicity).

As we experienced, students were motivated by these types of questions. It gave pupils a chance to make some experimentations, they experienced how to develop and extend a question, how to conjecture the answer, and how to ask new questions. We discuss these goals, the experiment itself, and the experiences in Section 7.

\section{Equations with complete characterization of solutions}

$$
\text { 2.1. Equation } \varphi(\Omega(n))=\Omega(\varphi(n))
$$

Theorem 2.1.

$\Omega(\varphi(n))=\varphi(\Omega(n))$ holds iff $n=2^{q}, n=3$, or $n=2^{\gamma} \cdot 3^{q-\gamma}$, where $q$ is a prime and $1 \leq \gamma \leq q-1$. 


\section{Proof}

Sufficiency

If $n=2^{q}$, then $\Omega\left(\varphi\left(2^{q}\right)\right)=\Omega\left(2^{q-1}\right)=q-1$, and $\varphi\left(\Omega\left(2^{q}\right)\right)=\varphi(q)=q-1$.

If $n=3$, then $\Omega(\varphi(3))=\Omega(2)=1$, and $\varphi(\Omega(3))=\varphi(1)=1$.

If $n=2^{\gamma} \cdot 3^{q-\gamma}$, where $q$ is a prime and $1 \leq \gamma \leq q-1$, then $\Omega\left(\varphi\left(2^{\gamma} \cdot 3^{q-\gamma}\right)\right)=$ $\Omega\left(2^{\gamma-1} \cdot 2 \cdot 3^{q-\gamma-1}\right)=q-1$, and $\varphi\left(\Omega\left(2^{\gamma} \cdot 3^{q-\gamma}\right)\right)=\varphi(q)=q-1$.

\section{Necessity}

First we show that no odd number $n>3$ can satisfy the equation.

(i) Let $3 \nmid n$, i.e. $n=\prod_{i=1}^{r} p_{i}^{\alpha_{i}}$, where $r \geq 1, p_{i}>3$ and $\alpha_{i} \geq 1$ for any $i \in \mathbb{Z}^{+}$.

On the right-hand side (further this will be denoted as RHS) we have $\varphi(\Omega(n))=\varphi\left(\sum_{i=1}^{r} \alpha_{i}\right) \leq\left(\sum_{i=1}^{r} \alpha_{i}\right)$, since $\varphi(k) \leq k$ for every $k \in \mathbb{Z}^{+}$.

On the left-hand side (further this will be denoted as LHS) we have $\Omega(\varphi(n)) \geq\left(\sum_{i=1}^{r}\left(\alpha_{i}-1\right)\right)+2 r=\left(\sum_{i=1}^{r} \alpha_{i}\right)+r$, since $\varphi(n)=\prod_{i=1}^{r} p_{i}{ }^{\alpha_{i}-1} \cdot\left(p_{i}-1\right)$, and $p_{i}-1$ is an even number with at least two prime factors, hence $\Omega\left(p_{i}-1\right) \geq 2$ for any $i \in \mathbb{Z}^{+}$.

So $\left(\sum_{i=1}^{r} \alpha_{i}\right)+r \leq \Omega(\varphi(n))=\varphi(\Omega(n)) \leq\left(\sum_{i=1}^{r} \alpha_{i}\right)$, a contradiction.

(ii) Consider now $3 \mid n$, where $n$ is not a power of 3 , i.e. $n=3^{\delta} \cdot \prod_{i=1}^{r} p_{i}{ }^{\alpha_{i}}$, where $\delta \geq 1, r \geq 1, p_{i}>3$ and $\alpha_{i} \geq 1$ for any $i \in \mathbb{Z}^{+}$.

RHS: $\varphi(\Omega(n))=\varphi\left(\delta+\left(\sum_{i=1}^{r} \alpha_{i}\right)\right) \leq \delta+\left(\sum_{i=1}^{r} \alpha_{i}\right)$.

LHS: $\Omega(\varphi(n)) \geq 1+\delta-1+\left(\sum_{i=1}^{r}\left(\alpha_{i}-1\right)\right)^{2}+2 r=\delta+\left(\sum_{i=1}^{r} \alpha_{i}\right)+r$, since $\Omega\left(\varphi\left(3^{\delta}\right)\right)=\Omega\left(2 \cdot 3^{\delta-1}\right)=1+\delta-1$.

So again the RHS is always less than the LHS.

(iii) If $n>3$ is a power of 3 , i.e. $n=3^{\delta}$, where $\delta>1$, then $\varphi\left(\Omega\left(3^{\delta}\right)\right)=$ $\varphi(\delta) \leq \delta-1$, but $\Omega\left(\varphi\left(3^{\delta}\right)\right)=\Omega\left(2 \cdot 3^{\delta-1}\right)=\delta$.

Now we turn to the case when $n$ is even. 
(iv) If $n>2$ is a power of 2 , i.e. $n=2^{\gamma}$, where $\gamma>1$, then $\varphi\left(\Omega\left(2^{\gamma}\right)\right)=\varphi(\gamma) \leq \gamma-1$, but $\Omega\left(\varphi\left(2^{\gamma}\right)\right)=\Omega\left(2^{\gamma-1}\right)=\gamma-1$, so equality holds iff $\gamma$ is a prime $q$, so $n=2^{q}$.

(v) Assume that $3 \nmid n$, but $n$ is not a power of 2, i.e. $n=2^{\gamma} \cdot \prod_{i=1}^{r} p_{i}^{\alpha_{i}}$, where $\gamma \geq 1, r \geq 1, p_{i}>3$ and $\alpha_{i} \geq 1$ for any $i \in \mathbb{Z}^{+}$.

RHS: $\varphi(\Omega(n)) \leq \gamma+\left(\sum_{i=1}^{r} \alpha_{i}\right)-1$.

LHS: $\Omega(\varphi(n)) \geq \gamma-1+\left(\sum_{i=1}^{r}\left(\alpha_{i}-1\right)\right)+2 r=\gamma+\left(\sum_{i=1}^{r} \alpha_{i}\right)+r-1$.

(vi) Next assume that $3 \mid n$ and $n$ has also a prime divisor greater than 3, i.e. $n=2^{\gamma} \cdot 3^{\delta} \cdot \prod_{i=1}^{r} p_{i}{ }^{\alpha_{i}}$, where $\gamma \geq 1, \delta \geq 1, r \geq 1, p_{i}>3$ and $\alpha_{i} \geq 1$ for any $i \in \mathbb{Z}^{+}$. RHS: $\varphi(\Omega(n)) \leq \delta+\gamma+\left(\sum_{i=1}^{r} \alpha_{i}\right)-1$.

LHS: $\Omega(\varphi(n)) \geq \gamma-1+\delta-1+1+\left(\sum_{i=1}^{r}\left(\alpha_{i}-1\right)\right)+2 r=\gamma+\delta+\left(\sum_{i=1}^{r} \alpha_{i}\right)+r-1$.

(vii) Finally consider $n=2^{\gamma} \cdot 3^{\delta}$, where $\gamma \geq 1, \delta \geq 1$. Since $\varphi(\Omega(n))=\varphi(\gamma+\delta) \leq$ $\gamma+\delta-1$ and $\Omega(\varphi(n))=\Omega\left(2^{\gamma-1} \cdot 2 \cdot 3^{\delta-1}\right)=\Omega\left(2^{\gamma} \cdot 3^{\delta-1}\right)=\gamma+\delta-1$, so equality holds iff $\gamma+\delta$ is a prime $q$, i.e. $n=2^{\gamma} \cdot 3^{q-\gamma}$.

\subsection{Equation $\Omega(\omega(n))=\omega(\Omega(n))$}

Theorem 2.2.

$\Omega(\omega(n))=\omega(\Omega(n))=k \Leftrightarrow n=\prod_{i=1}^{a} r_{i}^{\gamma_{i}}$ with $\sum_{i=1}^{a} \gamma_{i}=b$, where $r_{i}$ are distinct primes, $b \geq a$ are positive integers, and $\Omega(a)=\omega(b)=k$.

\section{Proof}

\section{$\frac{\text { Sufficiency }}{a}$}

If $n=\prod_{i=1}^{a} r_{i}{ }^{\gamma_{i}}$ satisfies the above conditions, then $\Omega(\omega(n))=\Omega(a)=k$ and $\omega(\Omega(n))=\omega(b)=k$, as well. 
Necessity

$\overline{\Omega(\omega(n))}=k$ holds iff $n=\prod_{i=1}^{a} p_{i}{ }^{\alpha_{i}}$, where $p_{i}$ are distinct primes with $\Omega(a)=k$. And $\omega(\Omega(n))=k \Leftrightarrow n=\prod_{j=1}^{s} q_{j}^{\beta_{j}}$, where $q_{j}$ are distinct primes with $\sum_{j=1}^{s} \beta_{j}=b$ and $\omega(b)=k$.

Hence the solutions of $\Omega(\omega(n))=\omega(\Omega(n))=k$ have to be of the form $n=\prod_{i=1}^{a} r_{i}^{\gamma_{i}}$ with $\sum_{i=1}^{a} \gamma_{i}=b$, where $\Omega(a)=\omega(b)=k$ and $b \geq a$.

This characterization also implies that we have infinitely many solutions in $n$ for every $k \geq 0$.

\section{Equations with infinitely many solutions}

In this section we deal with those equations, when though we cannot characterize all solutions but still we can give either infinitely many solutions in $n$ for every $k \in \mathcal{R}_{f} \cap \mathcal{R}_{g}$ of the equation $f(g(n))=g(f(n))=k$, or, being more modest, we can give infinitely many solutions in $n$ of $f(g(n))=g(f(n))$.

Theorem 3.1.

$\omega(d(n))=d(\omega(n))=k$ has infinitely many solutions for any $k \in \mathbb{Z}^{+}$.

\section{Proof}

$n=p_{1} \cdot p_{2}^{q_{1}-1} \cdot p_{3}^{q_{2}-1} \cdot \ldots \cdot p_{k}^{q_{k-1}-1} \cdot p_{k+1} \cdot \ldots \cdot p_{2^{k-1}}$ are solutions, where $p_{i}$ and $q_{j}>2$ are distinct primes, since $\omega(d(n))=\omega\left(d\left(2 \cdot q_{1} \cdot \ldots \cdot q_{k-1} \cdot 2^{2^{k-1}-k}\right)\right)=k$ and $d(\omega(n))=d\left(2^{k-1}\right)=k$.

Remark: in Section 7 we give a proof of one of the students which says that the equation has no composite solution which would be square free.

Theorem 3.2.

$\Omega(d(n))=d(\Omega(n))=k$ has infinitely many solutions for any $k \in \mathbb{Z}^{+}$.

\section{Proof}

$n=p_{1} \cdot p_{2}^{q^{k-1}-1}$ are solutions, where $p_{1} \neq p_{2}$ and $q$ are primes, as $\Omega(d(n))=\Omega\left(2 \cdot q^{k-1}\right)=k$ and $d(\Omega(n))=d\left(q^{k-1}\right)=k$. 
Theorem 3.3.

$\varphi(d(n))=d(\varphi(n))$ has infinitely many solutions.

Proof

$n=2^{p-1}$ are solutions, where $p$ is a prime, as $\varphi(d(n))=\varphi(p)=p-1$ and $d(\varphi(n))=d\left(2^{p-2}\right)=p-1$.

Also $n=2^{p-1} \cdot 3^{p-1}$ are solutions, where $p$ is a prime, as $\varphi(d(n))=\varphi\left(p^{2}\right)=$ $=(p-1) \cdot p$ and $d(\varphi(n))=d\left(2^{p-2} \cdot 2 \cdot 3^{p-2}\right)=d\left(2^{p-1} \cdot 3^{p-2}\right)=p \cdot(p-1)$.

Or $n=2^{2^{\alpha}-1} \cdot 3$ are solutions, where $\alpha \in \mathbb{Z}^{+}$, as $\varphi(d(n))=\varphi\left(2^{\alpha} \cdot 2\right)=\varphi\left(2^{\alpha+1}\right)=$ $=2^{\alpha}$ and $d(\varphi(n))=d\left(2^{2^{\alpha}-2} \cdot 2\right)=d\left(2^{2^{\alpha}-1}\right)=2^{\alpha}$.

Theorem 3.4.

$\varphi(\omega(n))=\omega(\varphi(n))=2$ has infinitely many solutions.

\section{Proof}

$n=2^{k} \cdot 3 \cdot 7$ are solutions, where $k$ is a positive integer, as $\varphi(\omega(n))=\varphi(3)=2$ and $\omega(\varphi(n))=\omega\left(2^{k-1} \cdot 2 \cdot 2 \cdot 3\right)=\omega\left(2^{k+1} \cdot 3\right)=2$.

4. Equations, where $f(g(n))=g(f(n))=k$ cannot be solved for all $k \in \mathcal{R}_{f} \cap \mathcal{R}_{g}$

Theorem 4.1.

Equation $\varphi(\sigma(n))=\sigma(\varphi(n))=2^{m}$ has no solutions for any $m \in \mathbb{Z}^{+}$.

Proof

Note that $\sigma(n)=2^{m} \Leftrightarrow n=\prod_{i} M_{p_{i}}$, where $M_{p_{i}}$ are distinct Mersenne primes (this determines the possible values of $m$, for which the $m$-th power of 2 is in the range of $\sigma(n))$.

This implies: $\sigma(\varphi(n))=2^{m} \Leftrightarrow \varphi(n)=\prod M_{p_{i}}$, a contradiction, since $\varphi(n)$ is even, if $n>2$, and also $\varphi(\sigma(2)) \neq \sigma(\varphi(2))$.

Theorem 4.2.

Equation $\sigma(d(n))=d(\sigma(n))=7$ has no solutions.

Proof

RHS: $\sigma(d(n))=7 \Leftrightarrow d(n)=4 \Leftrightarrow$ (i) $n=p_{1} \cdot p_{2}$, or (ii) $n=p^{3}$, where $p_{1} \neq p_{2}$ 
and $p$ are primes.

(i) If $n=p_{1} \cdot p_{2}$, then $d(\sigma(n))=d\left(\left(p_{1}+1\right) \cdot\left(p_{2}+1\right)\right)=7 \Leftrightarrow\left(p_{1}+1\right) \cdot\left(p_{2}+1\right)=q^{6}$. Since there is at least one odd prime amongst $p_{1}$ and $p_{2}, q=2$ must hold, which implies that $p_{1}$ and $p_{2}$ have to be Mersenne primes: $2^{2}-1,2^{3}-1$, or $2^{5}-1$. But the product $\left(p_{1}+1\right) \cdot\left(p_{2}+1\right)$ never equals $2^{6}$.

(ii) If $n=p^{3}$, then $d(\sigma(n))=d\left(p^{3}+p^{2}+p+1\right)=7 \Leftrightarrow p^{3}+p^{2}+p+1=q^{6} \Leftrightarrow$ $\frac{p^{4}-1}{p-1}=q^{6}$.

By [2], there is no solution of $\frac{x^{n}-1}{x-1}=y^{m}$, with integers $x, y>1$ and $m \geq 2$ if $n \equiv 0(4)$.

\section{Theorem 4.3.}

Equation $d(\varphi(n))=\varphi(d(n))=44$ has no solutions.

\section{Proof}

We enumerate all possible forms of $\varphi(n)$ determined by the RHS and the LHS, respectively, and show that there is no match.

RHS: $\varphi(d(n))=44$ holds iff
(A): $d(n)=3 \cdot 23$, or
(B): $d(n)=2^{2} \cdot 23$, or
(C): $d(n)=2 \cdot 3 \cdot 23$.

These allow the following forms of $n$ :
(A): $d(n)=3 \cdot 23 \Leftrightarrow(A 1) n=p^{68}$, or (A2) $n=p_{1}^{2} \cdot p_{2}^{22}$
(B): $d(n)=2^{2} \cdot 23 \Leftrightarrow$ (B1) $n=p^{91}$, or (B2) $n=p_{1}^{3} \cdot p_{2}^{22}$, or (B3) $n=p_{1} \cdot p_{2}^{45}$, or $(B 4) n=p_{1} \cdot p_{2} \cdot p_{3}^{22}$
(C): $d(n)=2 \cdot 3 \cdot 23 \Leftrightarrow(C 1) n=p^{137}$, or $(C 2) n=p_{1}^{5} \cdot p_{2}^{22}$, or (C3) $n=p_{1}^{2} \cdot p_{2}^{45}$, or (C4) $n=p_{1} \cdot p_{2}^{68}$, or (C5) $n=p_{1} \cdot p_{2}^{2} \cdot p_{3}^{22}$, where $p_{i}$ are distinct primes and $p$ is a prime.

These possible forms of $n$ induce the following values of $\varphi(n)$ :
(A1) $\varphi(n)=(p-1) \cdot p^{67}$, or $(A 2) \varphi(n)=\left(p_{1}-1\right) \cdot p_{1} \cdot\left(p_{2}-1\right) \cdot p_{2}^{21}$
(B1) $\varphi(n)=(p-1) \cdot p^{90}$, or $(B 2) \varphi(n)=\left(p_{1}-1\right) \cdot p_{1}^{2} \cdot\left(p_{2}-1\right) \cdot p_{2}^{21}$, or
(B3) $\varphi(n)=\left(p_{1}-1\right) \cdot\left(p_{2}-1\right) \cdot p_{2}^{44}$, 


$$
\begin{aligned}
& \text { or }(B 4) \varphi(n)=\left(p_{1}-1\right) \cdot\left(p_{2}-1\right) \cdot\left(p_{3}-1\right) \cdot p_{3}^{21} \\
& (C 1) \varphi(n)=(p-1) \cdot p^{136}, \text { or }(C 2) \varphi(n)=\left(p_{1}-1\right) \cdot p_{1}^{4} \cdot\left(p_{2}-1\right) \cdot p_{2}^{21}, \\
& \quad \text { or }(C 3) \varphi(n)=\left(p_{1}-1\right) \cdot p_{1} \cdot\left(p_{2}-1\right) \cdot p_{2}^{44}, \text { or }(C 4) \varphi(n)=\left(p_{1}-1\right) \cdot\left(p_{2}-1\right) \cdot p_{2}^{67}, \\
& \quad \text { or }(C 5) \varphi(n)=\left(p_{1}-1\right) \cdot\left(p_{2}-1\right) \cdot p_{2} \cdot\left(p_{3}-1\right) \cdot p_{3}^{21} .
\end{aligned}
$$

LHS: $d(\varphi(n))=44$ holds iff

$$
\text { (I): } \varphi(n)=q^{43} \text {, or }
$$

(II): $\varphi(n)=q_{1}^{3} \cdot q_{2}^{10}$, or

(III): $\varphi(n)=q_{1} \cdot q_{2}^{21}$, or

$(\boldsymbol{I V}): \varphi(n)=q_{1} \cdot q_{2} \cdot q_{3}^{10}$, where $q_{i}$ are distinct primes and $q$ is a prime.

Comparison:

(1) $n$ cannot be of the form $(A 1),(B 1),(B 3),(C 1),(C 3)$, or $(C 4)$, because no prime can have an exponent higher than 43 in the factorization of $\varphi(n)$.

(2) $n$ cannot be of the form $(B 2)$ or $(C 2)$, because there cannot be two distinct primes in the factorization of $\varphi(n)$, where one exponent is at least 21 , and the other is greater than 1.

(3) $n$ cannot be of the form $(A 2)$ or $(C 5)$, because $\varphi(n)$ cannot assume any of the listed forms $(I)-(I V)$.

(4) $n$ cannot be of the form $(B 4)$, because though $\varphi(n)=\left(p_{1}-1\right) \cdot\left(p_{2}-1\right)$. $\cdot\left(p_{3}-1\right) \cdot p_{3}^{21}$ could be of the form $\varphi(n)=q^{43}$ with $p_{3}=q=2$ and with $p_{1}, p_{2}$ being Fermat primes, but if $p_{1}=2^{a}+1$ and $p_{2}=2^{b}+1$ are primes with $a$ and $b$ being distinct powers of 2 , then $a+b=22$ should hold, and there are no such $a$ and $b$.

Remark: We can prove by similar arguments that $\varphi(d(n))=d(\varphi(n))=4 s$ has no solutions, if both $s$ and $2 s+1$ are primes and $2 s$ is not the sum of two powers of 2 less than 32. Similarly, $\varphi(d(n))=d(\varphi(n))=8 s$ has no solutions, if both $s$ and $4 s+1$ are primes and $4 s$ is not the sum of two powers of 2 less than 32 .

\section{Conditional results}

In this section we deal with the possibility of infinitely many solutions of the equation $f(g(n))=g(f(n))$ relying on some well known conjectures concerning primes. 
Proposition 5.1.

To any Mersenne prime $M_{p}$ there belongs a solution of equation $\omega(\sigma(n))=\sigma(\omega(n))$. The same holds for every prime of the form $p^{2}+p+1$, where also $p$ is a prime.

Proof

If $M_{p}=2^{p}-1$ is a Mersenne prime, then $n=2^{p-1}$ is a solution, since $\omega\left(\sigma\left(2^{p-1}\right)\right)=\omega\left(2^{p}-1\right)=\omega\left(M_{p}\right)=1$ and $\sigma\left(\omega\left(2^{p-1}\right)\right)=\sigma(1)=1$, as well.

If $q=p^{2}+p+1$ is a prime, where also $p$ is a prime (e.g. $p=3,5,17$ ), then $n=p^{2}$ is a solution, since $\omega\left(\sigma\left(p^{2}\right)\right)=\omega\left(p^{2}+p+1\right)=\omega(q)=1$ and $\sigma\left(\omega\left(p^{2}\right)\right)=\sigma(1)=1$, as well.

Remark: If there exist infinitely many Mersenne primes or infinitely many primes of the form $p^{2}+p+1$ with a prime $p$, then it follows from Proposition 5.1. that the equation $\omega(\sigma(n))=\sigma(\omega(n))$ has infinitely many solutions.

We note that Propositions 5.2.-5.3. indicate a similar statement.

\section{Proposition 5.2.}

For every odd prime of the form $q=\frac{p+1}{4}$, where also $p \neq 43$ is a prime there belongs a solution of equation $d(\sigma(n))=\sigma(d(n))$.

Remark: Though we know that there exist infinitely many primes $p$ in the arithmetic progression $a_{k}=4 k-1$, we do not know if this occurs infinitely many times with $k$ being a prime.

Proof

$n=3^{4} \cdot p$ is a solution if $p$ satisfies the above conditions, since $d\left(\sigma\left(3^{4} \cdot p\right)\right)=d\left(11^{2} \cdot(p+1)\right)=d\left(11^{2} \cdot 4 q\right)=d\left(2^{2} \cdot 11^{2} \cdot q\right)=18$, and $\sigma\left(d\left(3^{4} \cdot p\right)\right)=\sigma(10)=18$, as well.

\section{Proposition 5.3.}

For every prime of the form $q=\frac{p+1}{4}$, where also $p$ is a prime there belongs a solution of equation $\Omega(\sigma(n))=\sigma(\Omega(n))$.

\section{Proof}

$n=2^{2} \cdot p$ is a solution if $p$ satisfies the above conditions, since $\Omega\left(\sigma\left(2^{2} \cdot p\right)\right)=\Omega(7 \cdot(p+1))=\Omega(7 \cdot 4 q)=\Omega\left(2^{2} \cdot 7 \cdot q\right)=4$, and $\sigma\left(\Omega\left(2^{2} \cdot p\right)\right)=\sigma(3)=4$, indeed.

This leads us to a more general statement. 
Proposition 5.4.

If for all positive integer $l$ there exist primes $p>2$ and $q$ for which $q=\frac{p+1}{2^{l}}$ holds, then equation $\Omega(\sigma(n))=\sigma(\Omega(n))=k$ has at least one solution in $n$ for all $k$ in the range of function $\sigma$.

\section{Proof}

Let $n$ be of the form $n=2^{s} \cdot p$, where $p$ is an odd prime and $s$ is a positive integer. We need $\sigma\left(\Omega\left(2^{s} \cdot p\right)\right)=\sigma(s+1)=k$. This determines at least one value of $s$, since $k$ is in the range of function $\sigma$. On the other hand, $\Omega\left(\sigma\left(2^{s} \cdot p\right)\right)=\Omega\left(\left(2^{s+1}-1\right) \cdot(p+1)\right)=\Omega\left(2^{s+1}-1\right)+\Omega(p+1)$.

$\Omega\left(2^{s+1}-1\right)$ is determined by $s$, let it be $\Omega\left(2^{s+1}-1\right)=t$, i.e. we need $\Omega(p+1)=k-t$. Since $k \geq s+1$ and $\Omega\left(2^{s+1}-1\right) \leq \log _{2}\left(2^{s+1}-1\right)<s+1$, therefore $k-t \geq 1$ for any $s \in \mathbb{Z}^{+}$. If for all $k-t-1=l \in \mathbb{N}$ there exist primes $p, q$ for which $p+1=2^{l} \cdot q$ holds, then $n=2^{s} \cdot p$ is a solution, since $\Omega(\sigma(n))=\Omega\left(\left(2^{s+1}-1\right) \cdot(p+1)\right)=\Omega\left(2^{s+1}-1\right)+\Omega(p+1)=t+\Omega\left(2^{l} \cdot q\right)=$ $t+(l+1)=t+(k-t-1+1)=k$. Also $\sigma\left(\Omega\left(2^{s} \cdot p\right)\right)=\sigma(s+1)=k$, this determined the value of $s$.

For the next statement, we need the fact that to each Fermat prime $F_{i}$, $0 \leq i \leq 4$, there exists a prime $p_{i}$ such that $\frac{F_{i}^{p_{i}}-1}{F_{i}-1}$ are distinct primes; we can take e.g. $p_{0}=p_{1}=p_{2}=3, p_{3}=23$, and $p_{4}=7$.

\section{Proposition 5.5.}

Let $0 \leq k \leq 4$. Each set of Fermat primes $F_{i}, 0 \leq i \leq k$, with primes $p_{i}$, where $\frac{F_{i}^{p_{i}}-1}{F_{i}-1}$ are distinct primes, generates a solution of equation $\varphi(\sigma(n))=\sigma(\varphi(n))$.

Proof

$n=\prod_{i=0}^{k} F_{i}^{p_{i}-1}$, where $0 \leq k \leq 4$ is a solution if both $p_{i}$ and $\frac{F_{i}^{p_{i}}-1}{F_{i}-1}$ satisfy the above conditions.

RHS:

$$
\begin{aligned}
\varphi\left(\sigma\left(\prod_{i=0}^{k} F_{i}^{p_{i}-1}\right)\right) & =\varphi\left(\prod_{i=0}^{k} \frac{F_{i}^{p_{i}}-1}{F_{i}-1}\right)=\prod_{i=0}^{k}\left(\frac{F_{i}^{p_{i}}-1}{F_{i}-1}-1\right)= \\
& =\prod_{i=0}^{k} \frac{F_{i}^{p_{i}-1}-1}{F_{i}-1} \cdot \prod_{i=0}^{k} F_{i} .
\end{aligned}
$$


The last equality follows from $\frac{F_{i}^{p_{i}}-1}{F_{i}-1}-1=\frac{\left(F_{i}^{p_{i}-1}-1\right) \cdot F_{i}}{F_{i}-1}$.

LHS:

$$
\begin{aligned}
& \sigma\left(\varphi\left(\prod_{i=0}^{k} F_{i}^{p_{i}-1}\right)\right)=\sigma\left(\prod_{i=0}^{k} F_{i}^{p_{i}-2} \cdot\left(F_{i}-1\right)\right)=\sigma\left(\prod_{i=0}^{k} F_{i}^{p_{i}-2} \cdot 2^{2^{i}}\right)= \\
& =\sigma\left(\prod_{i=0}^{k} F_{i}^{p_{i}-2} \cdot \prod_{i=0}^{k} 2^{2^{i}}\right)=\sigma\left(\prod_{i=0}^{k} F_{i}^{p_{i}-2}\right) \cdot \sigma\left(\prod_{i=0}^{k} 2^{2^{i}}\right)= \\
& =\sigma\left(\prod_{i=0}^{k} F_{i}^{p_{i}-2}\right) \cdot \sigma\left(2^{2^{k}+2^{k-1}+\ldots+1}\right)=\prod_{i=0}^{k} \frac{F_{i}^{p_{i}-1}-1}{F_{i}-1} \cdot \sigma\left(2^{2^{k+1}-1}\right)= \\
& =\prod_{i=0}^{k} \frac{F_{i}^{p_{i}-1}-1}{F_{i}-1} \cdot\left(2^{2^{k+1}}-1\right)=\prod_{i=0}^{k} \frac{F_{i}^{p_{i}-1}-1}{F_{i}-1} \cdot\left(F_{k+1}-2\right)=\prod_{i=0}^{k} \frac{F_{i}^{p_{i}-1}-1}{F_{i}-1} \cdot \prod_{i=0}^{k} F_{i} .
\end{aligned}
$$

Remark: This is a generalization of the following observation by Golomb (see B42 in [3]): $n=3^{p_{0}-1}$ is a solution of $\varphi(\sigma(n))=\sigma(\varphi(n))$, if both $p_{0}$ and $\frac{3^{p_{0}}-1}{2}$ are primes.

Indeed, $\varphi\left(\sigma\left(3^{p_{0}-1}\right)\right)=\varphi\left(\frac{3^{p_{0}}-1}{2}\right)=\frac{3^{p_{0}}-3}{2}$, and $\sigma\left(\varphi\left(3^{p_{0}-1}\right)\right)=\sigma\left(2 \cdot 3^{p_{0}-2}\right)=$ $=3 \cdot\left(\frac{3^{p_{0}-1}-1}{2}\right)=\frac{3^{p_{0}}-3}{2}$ also. For $F_{0}=3$ such prime exponents are $p_{0}=$ $3,7,13,71$, and 103 .

In the case of $F_{1}=5$ and $F_{2}=17$ such prime exponent is e.g. $p_{1}=p_{2}=3$, hence $n=3^{6} \cdot 5^{2}$ and $n=3^{2} \cdot 5^{2} \cdot 17^{2}$ satisfy $\varphi(\sigma(n))=\sigma(\varphi(n))$.

Considering $F_{3}=257$ and $F_{4}=65537$, if we check the prime exponents with the help of WolframAlpha, it claims that for $F_{3}$ e.g. $p_{3}=23$, for $F_{4}$ e.g. $p_{4}=7$ works, so there are other solutions with four or five distinct prime factors, as well.

An open question is whether there exist infinitely many prime exponents $p_{i}$ for which $\frac{F_{i}^{p_{i}}-1}{F_{i}-1}$ are distinct primes, which would imply that equation $\varphi(\sigma(n))=$ $\sigma(\varphi(n))$ has infinitely many solutions. 


\section{Equation $g(f(n))=f(g(n))=1$}

This question can be solved easily in most cases, but it can also lead us to some unexpected difficulties, as well.

\section{Proposition 6.1.}

$\omega(\varphi(n))=\varphi(\omega(n))=1$ holds iff $n=F_{i}$, or $n=F_{i} \cdot F_{j}$, or $n=2^{\alpha}$, or $n=2^{\alpha} \cdot F_{i}$, where $\alpha \in \mathbb{Z}^{+}$and $F_{i} \neq F_{j}$ are Fermat primes.

Proof

$\varphi(\omega(n))=1 \Leftrightarrow(A) \omega(n)=1$ or $(B) \omega(n)=2$.

(A) $\omega(n)=1 \Leftrightarrow n=p^{\alpha}$, where $p$ is a prime and $\alpha \in \mathbb{Z}^{+}$.

Since $\omega\left(\varphi\left(p^{\alpha}\right)\right)=\omega\left((p-1) \cdot p^{\alpha-1}\right)=1 \Leftrightarrow(p-1) \cdot p^{\alpha-1}=q^{\gamma}$, where $q$ is a prime and $\gamma \in \mathbb{Z}^{+}$, this means:

(A1) in case of $\alpha=1$, then $p-1=q^{\gamma} \Leftrightarrow q=2$, so $p=2^{\gamma}+1$ is a prime, which implies $n=F_{i}$, or

(A2) in case of $\alpha>1$, then $p=2$, which implies $n=2^{\alpha}$.

(B) $\omega(n)=2 \Leftrightarrow n=p_{1}^{\alpha_{1}} \cdot p_{2}^{\alpha_{2}}$, where $p_{1} \neq p_{2}$ are primes and $\alpha_{1}, \alpha_{2} \in \mathbb{Z}^{+}$.

Since

$\omega\left(\varphi\left(p_{1}^{\alpha_{1}} \cdot p_{2}^{\alpha_{2}}\right)\right)=\omega\left(\left(p_{1}-1\right) \cdot p_{1}^{\alpha_{1}-1} \cdot\left(p_{2}-1\right) \cdot p_{2}^{\alpha_{2}-1}\right)=1 \Leftrightarrow$ $\left(p_{1}-1\right) \cdot p_{1}^{\alpha_{1}-1} \cdot\left(p_{2}-1\right) \cdot p_{2}^{\alpha_{2}-1}=q^{\gamma}$, where $q$ is a prime and $\gamma \in \mathbb{Z}^{+}$, this means:

(B1) in case of $\alpha_{1}>1, \alpha_{2}>1$, we have no solution,

(B2) if $\alpha_{1}>1, \alpha_{2}=1$, then $n$ has to be of the form $n=2^{\alpha} \cdot F_{i}$,

(B3) if $\alpha_{1}=1, \alpha_{2}=1$, then $n$ has to be of the form $n=F_{i} \cdot F_{j}$.

We leave the easy proofs of the following statements to the Reader:

Proposition 6.2.

(i) $\omega(d(n))=d(\omega(n))=1$ holds iff $n=p^{q^{\beta}-1}$, where $p, q$ are primes and $\beta \in \mathbb{Z}^{+}$.

(ii) $\Omega(d(n))=d(\Omega(n))=1$ holds iff $n=p$, where $p$ is a prime.

(iii) $\varphi(d(n))=d(\varphi(n))=1$ holds iff $n=1$ or $n=2$.

(iv) $\sigma(d(n))=d(\sigma(n))=1$ holds iff $n=1$. 
(v) $\varphi(\sigma(n))=\sigma(\varphi(n))=1$ holds iff $n=1$.

(vi) $\Omega(\sigma(n))=\sigma(\Omega(n))=1$ holds iff $n=2$.

(vii) $\Omega(\varphi(n))=\varphi(\Omega(n))=1$ holds iff $n=3,4$ or 6 . (See also Theorem 2.1.)

(viii) $\Omega(\omega(n))=\omega(\Omega(n))=1$ holds iff $n=\prod_{i=1}^{q} p_{i}{ }^{\alpha_{i}}$ and $\sum_{i=1}^{q} \alpha_{i}=r^{\beta}$, where $p_{i}$ are distinct primes, $q, r$ are primes, as well, and $\beta \in \mathbb{Z}^{+}$. (See also Theorem 2.2.)

\section{Problem 6.3.}

Finally, concerning $\omega(\sigma(n))=\sigma(\omega(n))=1$, we have only partial results. $\sigma(\omega(n))=1 \Leftrightarrow \omega(n)=1 \Leftrightarrow n=p^{\alpha}$, where $p$ is a prime and $\alpha \in \mathbb{Z}^{+}$. $\omega\left(\sigma\left(p^{\alpha}\right)\right)=\omega\left(\frac{p^{\alpha+1}-1}{p-1}\right)=1 \Leftrightarrow \frac{p^{\alpha+1}-1}{p-1}=q^{\beta}$, where $q$ is a prime and $\beta \in \mathbb{Z}^{+}$.

For $\beta=2$ it is known that the only solution is $p=3, \alpha=4$, and $q=11$ (see $[2])$. There are some more facts about the solvability of the more general NagellLjunggren equation $\frac{x^{n}-1}{x-1}=y^{m}$, where integers $x, y>1$ and $n>2, m \geq 2$, but these do not help to find solutions with $\beta>2$. It is not clear whether there exist such solutions. See Proposition 5.1. for some partial results with $\beta=1$.

\section{Why and how to teach this stuff?}

\subsection{Why?}

As we mentioned in the introduction, the concept of commutativity arises many times in high school maths, moreover, even earlier, in primary school maths. In the earliest years when children first meet positive integers and operations with them, they experience that addition and multiplication are commutative operations, however, subtraction and division are not (the same phenomenon appears with the associative law, as well). Later they learn about set-theoretical operations and realize that set-union, intersection, and symmetric difference are both commutative and associative, but these are false for the difference. In parallel with set-theoretical operations, pupils meet logical operations such as conjunction, 
disjunction, equivalence, and implication, and can decide which is commutative, and which is not. In geometry we do examine the commutative and associative laws when we teach vector operations (before we would teach that it is basically $\mathbb{R}^{2}$ ), and students experience that addition and dot product are commutative, subtraction and cross product are not (and addition is the only one amongst them which is associative). In special maths classes we teach matrix operations, as well, and it is a great opportunity to underline that though addition is commutative, multiplication does not have the same property (but both are associative). Finally, it will be clear in group theory that an operation being commutative is really not something evident and natural.

So there are many opportunities to clarify the meaning of a commutative operation, as it is an important concept, and in some cases we can even formulate a necessary and sufficient condition of commutativity in a proper situation.

One of these opportunities arises when we work with functions. Dealing with arithmetic functions might be the only opportunity to study discrete functions, as well. One might think that handling the problem of commutativity could be an easier question when working with discrete rather than with continuous functions, but as we saw in Sections 3-6, this is not the case. Still, having partial or conditional results, asking new questions, formulating conjectures, exploring the world of these kinds of problems can be challenging, adventurous, and useful, indeed, as we discuss it in detail in the next subsection.

\subsection{How?}

We present some details of the group study sessions held in this topic, highlight some experiences in a non-chronological order, share some thoughts about the goals, and add some didactic remarks, as well.

\section{The circumstances}

We had weekly one or two group study sessions with students in 9th grade. They learn in a special maths program, which means they have at least six regular maths classes weekly. Pupils have already learnt about arithmetic functions, and possess the basic knowledge in this topic. They did not have a routine in studying the composition of functions, but they needed just two examples to get the concept right and use it confidently. The first session was devoted to refresh memories (regarding definitions and formulas) and to rediscover some basic facts about the arithmetic functions in question. Right after this, we proposed the general problem to be studied, which students understood easily, and seemed to 
be motivated, enthusiastic, and eager to do some own research. Our goal was not to teach something, but to let them explore things on their own. Of course, the end of the school year being on the horizon, we did not expect them to do a lot of homework and to dedicate many hours to do research alone, so most of the explorations were guided by us. We planned the next session always based on the experiences of the previous one, so the content could reflect on what happened earlier. There were provisional plans for each class, but sometimes the kids' questions and ideas took an unexpected turn, and we tried to walk down the path they suggested rather than to follow the original scheme. The pupils got a summary of what happened in class (and also some questions to think about before the next meeting) via e-mail after each session.

\section{The sessions}

As students got a list of solutions $n<3000$ of the equation $\Omega(\varphi(n))=$ $\varphi(\Omega(n))$, and computed the standard form of these integers, it took them only a few minutes to come up with a conjecture concerning the general solution. We stated the theorem and verified the sufficiency in a joint work, but did not go through the details of proving the necessity in Theorem 2.1., because it would have taken too much time. We did discuss though that the proof would not require more than an exhaustive distinction of cases and relying on the trivial estimation $\varphi(m) \leq m$ valid for every $m \in \mathbb{Z}^{+}$. In later occasions, sometimes we proceeded similarly to save some time by giving the answer instead of asking them to find the solutions, but in other situations they did collect data themselves.

In Theorem 2.2. students suggested to examine the special case when $\Omega(n)=\omega(n)=q_{1} \cdot q_{2} \cdot \ldots \cdot q_{k}$ with distinct primes $q_{j}$. This implies $n=p_{1} \cdot p_{2} \cdot \ldots \cdot p_{q_{1} \cdot q_{2}} \cdot \ldots \cdot q_{k}$, where $p_{i}$-s are distinct primes. After they found this type of solutions, we discussed - through a proper example - how to find all solutions for a fixed $k$. Though they did not formulate the theorem in its most general form, giving a sufficient and necessary condition, they did understand the method how to find all solutions. We think, the emphasis was on the method itself, and so did not force to state a formalized theorem. Studying this question, students came up with some combinatorial ideas, as well. It was delightful to see how well they can connect different fields of maths and "think out of the box", indeed.

Comparing this latter theorem with the previously discussed Theorem 2.1., one of the goals was to point out the difference between the nature of the two answers. In Theorem 2.1. we can give all solutions in an explicit form, while in 
Theorem 2.2. we give a characterization of all solutions. The latter might be a bit unusual for students, but they should get used to it.

Turning to some problems in Section 3, we noticed that we might set up the subgoal of finding infinitely many solutions if we are unable to determine all solutions.

It was one of the highlights during these sessions when - right after the first session - a student proved that a composite integer satisfying $\omega(d(n))=d(\omega(n))$ in Theorem 3.1. cannot be square free. He did this on his own: he wanted to solve the equation, wrote a program in $\mathrm{C}++$ language, collected and analysed data, set up a conjecture, and proved it to be right. Here are his theorem and proof:

Theorem 7.2.1.

If $n$ is a composite solution of $\omega(d(n))=d(\omega(n))$, then there exists a prime $p$, for which $p^{2} \mid n$.

\section{Proof}

For a proof by contradiction, assume that $n=p_{1} \cdot p_{2} \cdot \ldots \cdot p_{k}$ with $k>1, k \in \mathbb{Z}^{+}$ and distinct primes $p_{i}$.

On the LHS we have $\omega\left(2^{k}\right)=1$, but the RHS is $d(k)$, which is a contradiction, since the RHS must be greater than 1 , if $k>1, k \in \mathbb{Z}^{+}$.

Though his original aim was different, he did notice something and was able to prove it. Also, it is very useful to deal not just with constructive or existence proofs, but also with this type of "non-existence" proofs, when we show that something is impossible.

Considering a particular case of Theorem 3.2., some students were searching solutions among the powers of 2 . The reason for this might have been that we had dealt with some problems before, when the powers of 2 played a useful role, and encouraged by these experiences, they wanted to check whether we could easily find some solutions in this case, as well. They proved that $n=2^{\alpha}$ is a solution of the equation $\Omega(d(n))=d(\Omega(n))=2$ iff $\alpha$ is a prime such that also $\frac{\alpha+1}{2}$ is a prime.

We mention Theorem 3.3. just to demonstrate that though students identified a lot of patterns right, of course they did have some false thoughts, as well. But it is a self-evident part of the thinking process, and as teachers we think that it was good and important for students to experience that the first idea is not always the 
right one, and this is not a problem at all. So they got that the first twenty solutions of the equation $\varphi(d(n))=d(\varphi(n))$ are 1, 2, 4, 6, 16, 24, 30, 36, 64, 384, 408, $480,510,1024,1296,1560,1680,2304,2640,3480$, and they computed the standard form of these integers. Considering the powers of 2, first someone conjectured that the exponents greater than one $(2,4,6)$ are the consecutive terms in the sequence of even numbers. This turned out to be wrong, because $2^{8}$ is not a solution. There was another suggestion upon the exponents $2,4,6,10$, that these would satisfy a Fibonacci type recursion. This proved to be wrong, as well, since $2^{26}$ is not a solution (but $2^{12}$ is one). After discussing these false conjectures, we proved that a power of 2 is a solution of the equation if and only if the exponent is by one less than a prime. We cherish these kinds of brainstorming though, and think that kids can learn a lot also from the mistakes. But of course, it is great if they can find the right answer at the end.

We did not examine any of the problems in Section 4, though we discussed all the details to prove Theorem 4.1. We think, however, that while studying Theorem 4.1. for instance (or constructing solutions in Proposition 5.1. and in Proposition 5.5., or solving Proposition 6.1.), it is important for pupils to experience that those "famous" primes they learned about in class can play a role in their own work and not just in history books.

While chasing solutions, the question arises naturally whether there is at least one suitable value $k$ in the range of both functions $f$ and $g$ for which the equation $f(g(n))=g(f(n))=k$ is not solvable. We used the well known problem solving strategy in Theorem 4.3.: if we have a problem and the set of possible solutions (in this case: the set of standard forms of potential integers $n$ ) is finite, then we "just" have to enumerate all possibilities and figure out if there exist solutions among them or not. We use this kind of strategy in various situations in our math studies, so this might be a familiar way for pupils. While we focused on proving that there exists no solution in a particular case, we could try to make some generalizations. We got some results of this type in the remark after the proof of Theorem 4.3.

There are, of course, some situations when we cannot expect the answer from the students. For example, part (ii) in the proof of Theorem 4.2. relies on a deep theorem far beyond the reach of the students, but we can still tell them about this result, as we did in a class when we talked about the propositions of Section 6 .

We have some conditional results in Section 5. We think that it is really useful to talk about these kinds of issues, since there are many such important 
results in mathematics, e.g. lots of papers begin with: "Suppose the Riemann hypothesis is true...". From this section we went through the proof of Proposition 5.5. In a way it was a little summary of the research: we formulate the question, read some facts about it in the existing literature, use some software to collect data, organize these data, identify some patterns, conjecture some more general solutions than given in [3], and prove them to be right by using the well known recursion for these primes. It was another great opportunity to demonstrate that these "famous" primes can actually play a role in our "everyday" problem solving, and it also gave us a chance to connect seemingly remote parts of mathematics. We discussed this theorem in the last session and shared some personal thoughts on how a new result can be born.

Finally we mention, that it was useful to investigate also the less complex problems of Section 6 during an earlier session. This gave the students some self-confidence, as they could verify Proposition 6.2. without facing any major difficulty. It was a bit harder to deal with Proposition 6.1. where it is not that difficult to set a conjecture but the proof required a thorough examination, one of the pupils got even somewhat lost, but finally could get through with some help. Anyway, the above proofs were accessible and easily understandable for the students. It was interesting to see after solving these equations more or less easily, that the last variant Proposition 6.3. - which played a role also in the proof of Theorem 4.2. - is an unsolved problem. This makes clear that there is definitely plenty of room for more research, and there are many open questions in this topic, as well.

\subsection{New horizons to be conquered}

There are many directions to ask entirely new questions or to generalize some previously obtained results. We show a few such examples below.

7.3.1. The following natural question arises: can we define pairs of functions $f$ and $g$, where $f(g(n))=g(f(n))$ holds for all $n \in \mathbb{Z}^{+}$? This clearly holds if $f=g$, or $g$ is the identity. The good students can find the general scheme $f=h^{(k)}$ and $g=h^{(l)}$, where $h^{(m)}$ means the composition of $m$ copies of $h$. But there are many other examples, as well; students might come up with a solution, like:

$$
f(n):= \begin{cases}1 & \text { if } n \text { is even; } \\ 2 & \text { if } n \text { is odd. }\end{cases}
$$


and

$$
g(n):=\left\{\begin{array}{lc}
n & \text { if } n \leq 2 ; \\
2 k & \text { if } n>2 \text { is even; } \\
2 m-1 & \text { if } n>2 \text { is odd. }
\end{array}\right.
$$

where $k$ and $m$ are fixed positive integers.

We can ask also the other extreme situation, when $f(g(n))=g(f(n))$ does not hold for any $n \in \mathbb{Z}^{+}$, except perhaps for $n=1$.

7.3.2. In Theorem 3.4. we studied equation $\varphi(\omega(n))=\omega(\varphi(n))=2$. Can we find infinitely many solutions in $n$ of $\varphi(\omega(n))=\omega(\varphi(n))=k$ for every $k \in \mathcal{R}_{f} \cap \mathcal{R}_{g}$ ?

Let's consider e.g. $k=8$. Equation $\varphi(\omega(n))=8$ holds iff $\omega(n)=15,16,20$, 24 , 30. Choosing e.g. $\omega(n)=15$, i.e. $n$ is of the form $n=p_{1}^{\alpha_{1}} \cdot p_{2}^{\alpha_{2}} \cdot \ldots \cdot p_{15}^{\alpha_{15}}$, where primes $p_{i}$ are distinct, can we give the suitable values of primes $p_{i}$ and exponents $\alpha_{i}$, so $\omega(\varphi(n))=8$ holds, as well?

First - as we use this problem solving strategy in class, too - we simplify and specialize the problem, and search for solutions if $p_{1}=2, \alpha_{1} \geq 1$ and $\alpha_{2}=\alpha_{3}=$ $\ldots=\alpha_{15}=1$, so $n$ is of the form $n=2^{\alpha_{1}} \cdot p_{2} \cdot \ldots \cdot p_{15}$, for which the product $\varphi(n)=2^{\alpha_{1}-1} \cdot\left(p_{2}-1\right) \cdot \ldots \cdot\left(p_{15}-1\right)$ has to have eight distinct prime factors.

Let primes $p_{i}$ be defined as follows: $p_{2}=2^{\beta_{1}}+1, p_{3}=2^{\beta_{2}} \cdot 3^{\gamma_{1}}+1$, $p_{4}=2^{\beta_{3}} \cdot 5^{\delta_{1}}+1, \ldots, p_{8}=2^{\beta_{7}} \cdot 17^{\eta_{1}}+1$, and $p_{9}=2^{\beta_{8}} \cdot 19^{\zeta_{1}}+1$, where all the exponents (from the Greek alphabet) are positive integers, so the product of the factors $p_{i}-1$ with $1 \leq i \leq 9$ contains eight distinct primes. Now we have to make sure that the factors $p_{i}$ with $10 \leq i \leq 15$ do not add a new prime factor into $\varphi(n)$. So let: $p_{i}=2^{a_{i}} \cdot 3^{b_{i}} \cdot 5^{c_{i}} \cdot 7^{d_{i}} \cdot 11^{e_{i}} \cdot 13^{f_{i}} \cdot 17^{g_{i}} \cdot 19^{h_{i}}+1$, where $a_{i} \geq 1$ and the other exponents (from the Latin alphabet) in $p_{i}$ are non-negative integers. There exist such primes, e.g. $p_{2}=3, p_{3}=7, p_{4}=11, p_{5}=29, p_{6}=23, p_{7}=53, p_{8}=137, p_{9}=1217$ and $p_{10}=5, p_{11}=17, p_{12}=257, p_{13}=65537, p_{14}=13, p_{15}=97$.

This construction would work for any value in the range of $\varphi$ - which would also mean that there are infinitely many solutions in $n$ with $\alpha_{1}$ being arbitrary large - if there is at least one prime in classes of the first type and if there are "some more" in some classes, as well.

7.3.3. In Theorem 4.1. we showed that the equation $\varphi(\sigma(n))=\sigma(\varphi(n))=k$ cannot be solved for every $k \in \mathcal{R}_{\varphi} \cap \mathcal{R}_{\sigma}$, since there is no solution if $k$ is a power of 2 . There are other values in the range of both functions for which the 
equation has no solution. E.g. $k=6$ is in the range, i.e. $\sigma(5)=6=\varphi(7)$, but $\varphi(\sigma(n))=\sigma(\varphi(n))=6$ has no solution, because $\sigma(\varphi(n))=6$ could hold only if $\varphi(n)=5$, which is a contradiction, since $\varphi(n)$ is even, if $\varphi(n)>1$.

Generalizing this question, we can study the solvability of $\varphi(\sigma(n))=$ $=\sigma(\varphi(n))=p+1$, where $p$ and $p+2$ are twin primes, like 5 and 7 in the previous example. The values of $p+1$ are in the range of both functions, since $\sigma(p)=p+1$ and $p+1=\varphi(p+2)$, as well, with $p$ and $p+2$ being primes. The next question is if there exists a solution when $p+1=12$ ? It is necessary that $\varphi(n)=6$ or $\varphi(n)=11$. Latter is a contradiction, and since $\varphi(n)=6$ holds iff $n=7,9,14$, or 18 , we have to check only four possible values of $n$ whether $\varphi(\sigma(n))=12$ also holds. As it turns out, $n=9$ is the only solution, so $\varphi(\sigma(n))=\sigma(\varphi(n))=12$ holds iff $n=9$. In Proposition 5.5. we claimed that $n=3^{2}$ is a solution of $\varphi(\sigma(n))=\sigma(\varphi(n))$, now we have that it is the only solution of $\varphi(\sigma(n))=\sigma(\varphi(n))=12$.

Examining the equation $\varphi(\sigma(n))=\sigma(\varphi(n))=p+1$, where $p$ and $p+2$ are twin primes, we find that values of $p+1$ for which there is a solution, occur rarely, since there are only two among the first 40 twin primes: $p+1=12$ and $p+1=1092$. The latter we have if $n=3^{6}$, also mentioned in Proposition 5.5., and it is also the only solution of $\varphi(\sigma(n))=\sigma(\varphi(n))=1092$.

How can students study these questions? Here we present a way of it, but it is definitely not the only one. If pupils want to know if there exists a solution for example when $p+1=42$, they can use GeoGebra to get the possible values of $\varphi(n)$ for which $\sigma(\varphi(n))=42$. They can find that $\sigma(m)=42$ holds iff $m=20,26$, or 41. Of course, students could get these answers without any help, just calculating these, but the aim here is not to exercise "inverting" function $\sigma$, but to solve the original equation. Since $\varphi(n)$ is even, we only have to examine the solutions of $m=\varphi(n)=20$ and $m=\varphi(n)=26$, respectively. At this point we can use WolframAlpha to get the values of $n$, for which these equations hold. Equation $\varphi(n)=20$ holds iff $n=25,33,44,50$, or 66 , equation $\varphi(n)=26$ has no solution. So we have five possible values of $n$, for which we have to check (using GeoGebra again) if $\varphi(\sigma(n))=42$ holds, as well, and we get that none of the above listed values satisfy the equation.

Thus students will probably conclude that it is pretty hard to get solutions of this type (if there are more such solutions at all), but they should feel that it was nice to give a try to this promising idea and should not consider it as a fiasco despite of the modest results. And we can encourage them to find new paths to explore new horizons, this is how research is done. 
I am very grateful for the help and suggestions of my supervisor, Róbert Freud and for the referees' valuable remarks. And very special thanks to the students of 9.b in the high school Óbudai Árpád Gimnázium for their dedication and enthusiasm, and also thanks to their mathematics teacher who made this experiment possible.

\section{References}

[1] R. Freud, E. Gyarmati, Számelmélet, Nemzeti Tankönyvkiadó, Budapest (in Hungarian), 2000.

[2] Y. Bugeaud, P. Mihailescu, On the Nagell-Ljunggren equation $\frac{x^{n}-1}{x-1}=y^{q}$, Mathematica Scandinavica 101 (2007), 177-183.

[3] R.K. Guy, Unsolved Problems in Number Theory, 2nd edition, Springer-Verlag, New-York, 1994, 99.

[4] J. Sándor, On the composition of some arithmetic functions, Studia Univ. Babes-Bolyai Mathematica 34 (1989), 7-14.

[5] J. Sándor, On the composition of some arithmetic functions, II, Journal of Inequalities in Pure and Applied Mathematics 6, no. 3 (2005), 73.

[6] C. Pomerance, On the composition of the arithmetic functions $\varphi$ and $\sigma$, Colloquium Mathematicum 58 (1989), 11-15.

[7] F. Luca, C. Pomerance, On some problems of Makowski-Schinzel and Erdős concerning the arithmetic functions $\phi$ and $\sigma$, Colloquium Mathematicum 92 (2002), 111-130.

[8] Shukla D.P., Yadav Shikha, Composition of arithmetical functions with generalization of perfect and related numbers, Commentationes Mathematicae 52, no. 2 (2012), 153-170.

ILDIKÓ KÉZÉR

ÓBUDAI ÁRPÁD GIMNÁZIUM, BUDAPEST, HUNGARY

1034 BUDAPEST NAGYSZOMBAT UTCA 19.

E-mail: kezerildi@gmail.com

(Received December, 2017) 